\title{
Bio-inspired computing for hybrid information technology
}

\author{
Binod Vaidya · Jong Hyuk Park $\cdot$ Hamid R. Arabnia • \\ Witold Pedrycz $\cdot$ Sheng-Lung Peng
}

Published online: 3 June 2011

(c) Springer-Verlag 2011

Recently biologically inspired, converged and hybrid information technologies have become of significant interest due to their capabilities in handling many real world complex problems, involving imprecision, uncertainty and vagueness and highdimensionality. This special issue is committed to capture the state-of-the-art and recent advances on various aspects of biologically inspired computing for hybrid information technology. It aims to bring together the researchers from academia and industry as well as practitioners to share ideas, problems and solutions relating to the multifaceted aspects of information technology, especially focused bio-inspired computing. Original articles describing algorithms, protocols, performance evaluations, and case studies on the bio-inspired hybrid information technologies are solicited for this special issue.

Topics include, but not limited to:

- Bio-inspired information and communication systems

B. Vaidya

Institute of Telecommunications, Covilha, Portugal

e-mail: bnvaidya@gmail.com

J. H. Park $(\square)$

Seoul National University of Science and Technology,

Seoul, Korea

e-mail: parkjonghyuk1@ hotmail.com

H. R. Arabnia

The University of Georgia, Athens, USA

e-mail: hra@cs.uga.edu

W. Pedrycz

University of Alberta, Edmonton, Canada

e-mail: pedrycz@ee.ualberta.ca

S.-L. Peng

National Dong Hwa University, Hualien, Taiwan

e-mail: slpeng@mail.ndhu.edu.tw
- Bio-inspired networking for pervasive computing environments

- Bio-inspired mathematical models, methods and tools

- Bio-inspired network and communication algorithms and protocols

- Biologically inspired fault-tolerant communication systems

- Cluster and grid implementation of bio-inspired networks.

- Self-organizing, self-learning and self-healing network architectures

- Novel applications and services inspired by biological systems

- Scalable and adaptive network architectures

- Performance evaluation of bio-inspired networks

- Bio-inspired mechanisms for network and information security

- Bio-inspired intrusion-tolerant systems

- Biologically inspired and cognitive strategies for cyber defense

- Bio-inspired service evolution and optimization

- Topology control and network organization for bioinspired networks

- Bio-inspired localization, synchronization and mobility approaches

- Nano-scale and molecular computing and communications

- Case studies of biologically inspired networks.

We received 15 manuscripts. Each manuscript was blindly reviewed by at least three reviewers consisting of guest editors and external reviewers. After the review process, eight manuscripts were finally selected for this Special Issue.

The first paper in this special issue is on Wavelets Based Facial Expression Recognition Using a Bank of Support 
Vector Machines, by Sidra Batool Kazmi, Qurat-ul-Ain, M. Arfan Jaffar. This paper proposes a method for automatic facial expression recognition. It finds out the face region and extracted the reduced features using 2-D wavelet and PCA. These features are supplied to a bank of seven binary support vector machines. The testing is performed 100 times and the promising average accuracies for facial expression recognition ranging from 81.67 to $96.00 \%$ have been achieved by the proposed method.

The second paper in this special issue is on Putative Xylosyltransferase Genes in Trichomonas vaginalis, by Kuo-Yuan Hwa, Boopathi Subramani and Kay-Hooi Khoo. Xylosyltransferase (XT) genes are identified in a unicellular organism $T$. vaginalis through in silico approach based on the sequence homology. To confirm if these genes are putative XT genes, a workflow is designed based on the sequence characteristics of glycosyltransferase, with PfamHMM, TMHMM and MEME. The results confirm that these XTs are related to N-linked XTs in plant cells. In addition, the paper analyzes the N-linked glycans of $T$. vaginalis. The empirical data also supported the computational analysis.

The third paper in this special issue is on LARGE like GlcNAc Transferase Database (LGTBase), by Kuo-Yuan Hwa, Wan Man Li, Chueh-Pai, Li. The paper describes an intelligent computational platform for the LGTBase, by setting up an automatic updating databank for genes of the LARGE family and by integrating bioinformatics tools. The new platform can assist biologists to obtain the most update information about the LARGE family and to predict if a gene was a member of the LARGE family.

The fourth paper in this special issue is on Effects of 12-week Circuit Weight Training and Aerobic Exercise on Body Composition, Physical Fitness, and Pulse Wave Velocity in Obese Collegiate Women, by Hyun-Joo Kang, Yang Sun Lee, Doo-Soon Park, Duk-Ho Kang. This paper studies the effects of 12 weeks of exercise on body composition, physical fitness. Pulse wave velocity in obese collegiate women and circuit weight training and aerobic exercise have favorable effects on the occurrence of obesity and physical fitness in obese collegiate women.

The fifth paper in this special issue is on SignatureClust: A Tool for Landmark Gene-guided Clustering, by Pankaj Chopra, Hanjun Shin, Jaewoo Kang, Sunwon Lee. This paper introduces a new software package, SignatureClust, for clustering gene expression data. SignatureClust allows users to select a group of functionally related genes (called 'Landmark Genes'), and to project the gene expression data onto these genes. Compared to existing algorithms and software in this domain, the proposed software package offers an important benefit. By selecting different sets of landmark genes, it enables the user to cluster the microarray data from multiple biological perspectives, and thereby allows the users to explore and interpret the data from multiple different aspects.

The sixth paper in this special issue is on Termite Tunneling Feature Extraction Using Genetic Algorithm, by Eun-Kwan Kang, Malrey Lee and Thomas Gatton. Path design uses information about termite reproduction and the termite environment necessary for tunneling. Features are extracted by analyzing relevance of this information, and the fitness and relevance of these features are evaluated. The proposed method in this paper is capable of finding various optimal termite tunneling paths.

The seventh paper in this special issue is on Distributed Quantum Entanglement Sharing (DQES) Model for Highperformance Real-time System, by Chi-Yuan Chen, YaoHsin Chou, Han-Chieh Chao. In this paper, they propose the DQES Model to share quantum entanglement with processors. They also present some possible applications such like database consistency, jobs scheduling, system dependability and reliable communication protocols.

The last paper in this special issue is on Bio-Inspired Group Mobility Model for Mobile Ad Hoc Networks Based on Bird Flocking Behavior, by Sudip Misra, Prateek Agarwal. This paper proposes a novel group mobility model for mobile ad hoc networks (MANETs), named as Bird Flocking Behavior Inspired Group Mobility Model (BFBIGM), which takes inspiration from the mobility of a flock of birds, flying in a formation. In the proposed approach, the superposition of a simple set of flocking rules, cohesion, following, homing, dispersion and alignment, on each individual node gives a stable formation flight at any velocity and altitude. The paper also runs different simulations to compare the performance of the proposed model with the Random Way Point and the Reference Point Group Mobility (RPGM) models by considering some mobility and connectivity metrics. The simulation results show that the proposed mobility model is highly spatially correlated and has higher link duration among MNs, as compared to other group model such as RPGM.

Finally, we would like to thank all authors for their contributions to this special issue. We also extend our thanks to the following external reviewers for their excellent job in reviewing the manuscripts: Wei Chen, Stefanos Gritzalis, Zheng Yan, Leonard Barolli, Doo-soon Park, Sang Oh Park, Kyusuk Han, Naveen Chilamkurti, Damien Sauveron, Jonghyuk Lee, Jongsung Kim, Naixue Xiong, Changhoon Lee, Yangsun Lee, Isaac Woungang, Sang-Soo Yeo, Deok Gyu Lee, Taeshik Shon, Hangbae Chang and Seungmin Rho. 